\title{
Angiosarcoma of the Head and Neck
}

\author{
Brandon Mullins ${ }^{1}$ Trevor Hackman ${ }^{1}$ \\ ${ }^{1}$ Department of Otolaryngology/Head and Neck Oncology, University \\ of North Carolina Hospitals, Chapel Hill, North Carolina, United States \\ Int Arch Otorhinolaryngol 2015;19:191-195.
}

\begin{abstract}
Address for correspondence Brandon Mullins, MSc, University of North Carolina at Chapel Hill - School of Medicine, 1021 Dawes St, Chapel Hill, North Carolina 27516, United State

(e-mail: brandon_mullins@med.unc.edu).
\end{abstract}

\begin{abstract}
Keywords

- angiosarcoma

- head and neck neoplasms

- radiotherapy

- surgery

Introduction Angiosarcoma of the head and neck is a rare vascular sarcoma associated with high rates of local recurrence and distant metastasis and a poor prognosis.

Objective We describe our experience treating patients with angiosarcoma of the head and neck to evaluate the outcomes, patterns of failure, and current treatments. Methods We identified six patients with angiosarcoma of the head and neck and treated at our institution between 2000 and 2013. We compared our results to the literature from 1979 to 2013.

Results Mean follow-up was 42 months. Local recurrence rate was $50 \%$ with diseasespecific survival and 2-year disease-free survival rates of 33.3 and $20 \%$, respectively. Prognostic factors included tumor size $>5 \mathrm{~cm}$ and surgical margin status, with no correlation between histologic grade and survival. Combined-modality therapy was only used for aggressive tumors with positive surgical margins but is suggested to improve local control and overall survival.

Conclusions Our data series supports that angiosarcoma of the head and neck has a high rate of recurrence and is associated with a poor prognosis, despite current combined-modality therapy. The study highlights the importance of attaining negative margins during surgical resection, the utility of adjuvant therapies, as well as the need for continued research in developing new management strategies.
\end{abstract}

\section{Introduction}

Angiosarcoma is a rare vascular neoplasm arising from endothelial cells and accounting for less than $2 \%$ of soft tissue sarcomas and less than $1 \%$ of all head and neck malignancies. ${ }^{1-3}$ It can occur in any part of the body, but the head and neck variety commonly affects the scalp and face of individuals over the age of $60 .^{1-4}$ It more commonly affects males than females and is slightly more common in Caucasian patients. ${ }^{1,4}$ Historically, angiosarcoma is a very aggressive neoplasm with high rates of local recurrence, early metastases, and a poor prognosis. Our goal is to add to the literature by reporting the clinical presentation, prognostic factors, and outcomes from our single institutional study with a literature review.

received

September 5, 2014

accepted

January 10, 2015

published online

March 10, 2015

\section{Methods}

This Institutional Review Board-approved retrospective series describes six patients with angiosarcoma of the head and neck treated at our institution. They were diagnosed based on a thorough history and physical examination, and disease was pathologically confirmed through biopsy. Treatment methods included surgical excision, radiation therapy, chemotherapy, or a multimodality approach.

\section{Results}

In our review, we identified six patients with angiosarcoma of the head and neck during a 13-year period (2000 to 2013). The demographics and clinical characteristics of the patients

Copyright $\odot 2015$ by Thieme Publicações License terms Ltda, Rio de Janeiro, Brazil
DOI http://dx.doi.org/ 10.1055/s-0035-1547520. ISSN $1809-9777$.

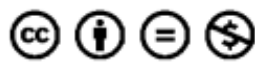


are summarized in - Table 1. All patients are from the United States. The mean age of the patients was 65.7 (range 52 to 83) with most of the patients being male (5 to 1 ). Presenting signs and symptoms included an itching or bleeding lesion (33.3\%), pain at the lesion site (33.3\%), cough (16.7\%), bloody sputum (16.7\%), or epistaxis (16.7\%), depending on the tumor location. The most common presenting symptom was an enlarging mass (83.3\%).

During diagnosis, all patients had a thorough clinical workup, physical examination, and either computed tomography or magnetic resonance imaging of the tumor. On imaging, tumor sizes ranged from 2.2 to $7.5 \mathrm{~cm}$. Tumors were biopsied for final pathologic confirmation (-Figs. 1 and 2 ).

Pathologic tumor size and grade were reported for most patients within the series. The mean tumor size was $5.54 \mathrm{~cm}$ (range 2.2 to $7.5 \mathrm{~cm}$ ). Actual tumor sizes for patients 2, 5, and 6 were larger than reported as they all contained multiple positive surgical margins on excision. There was no reported precise tumor size for patient 3, but the tumor was reported as large and unresectable, extending throughout the larynx and hypopharynx. For pathologic grading, patient 1 had low-grade tumor, patients 3 and 4 had intermediate-grade tumors, and patients 2, 5, and 6 had high-grade tumors.

For treatment intervention, which is summarized in -Table 2, 4 patients had tumors treated with curative intent and 2 patients received palliative treatment (patients 3 and 5), based on difficulty of surgical removal. All 4 patients treated with curative intent received surgical intervention. Three of the four patients had positive postsurgical margins (patients 1, 2, and 6), and those 3 patients also received radiation therapy either pre- or postsurgery. Patient 6 was the only patient to receive neoadjuvant chemotherapy (weekly Taxol ${ }^{\circledR}$, Bristol-Myers Squibb, NY, USA) and radiation therapy prior to surgical resection. For palliative treatment, patient 3 received chemotherapy (weekly Taxol) and patient 5 received radiation therapy.

Patient were followed for an average period of 42 months. Clinical outcomes are also summarized in - Table 2. Two of the four surgical patients (50\%) had disease recurrence. One patient had a local recurrence (patient 1), and 1 showed

Table 1 Clinical characteristics of the angiosarcoma series

\begin{tabular}{|c|c|c|c|c|c|c|}
\hline Case & Age & Gender & Race & Location & Tumor size $(\mathrm{cm})$ & Symptoms \\
\hline 1 & 71 & Male & African American & Left cheek & 4.5 & Enlarging mass \\
\hline 2 & 76 & Female & African American & Scalp & 6.8 & Itchy and bleeding lesion \\
\hline 3 & 55 & Male & Caucasian & Larynx and hypopharynx & $\begin{array}{l}\text { No size listed; reported } \\
\text { as extensive throughout } \\
\text { larynx and hypopharynx }\end{array}$ & $\begin{array}{l}\text { Neck pain, enlarging } \\
\text { mass, cough and bloody } \\
\text { sputum }\end{array}$ \\
\hline 4 & 52 & Male & Caucasian & Maxillary sinus & 2.2 & $\begin{array}{l}\text { Epistaxis, facial pain and } \\
\text { enlarging mass. }\end{array}$ \\
\hline 5 & 83 & Male & Caucasian & Scalp & 7.5 & $\begin{array}{l}\text { Bleeding and enlarging } \\
\text { mass }\end{array}$ \\
\hline 6 & 57 & Male & Caucasian & Left cheek & 6.7 & Enlarging mass \\
\hline
\end{tabular}

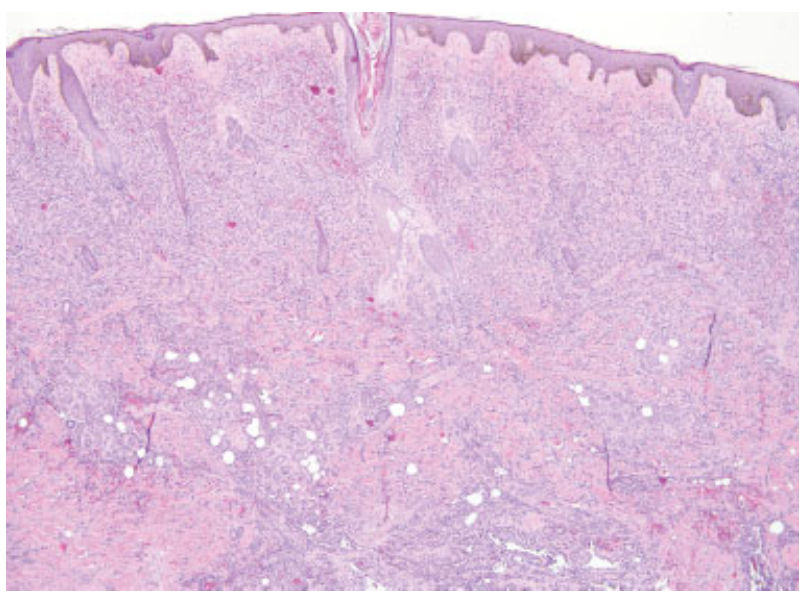

Fig. 1 Hematoxylin and eosin staining of a tumor section of patient 2 demonstrating extensive dermal involvement by angiosarcoma $(\times 40$ magnification).

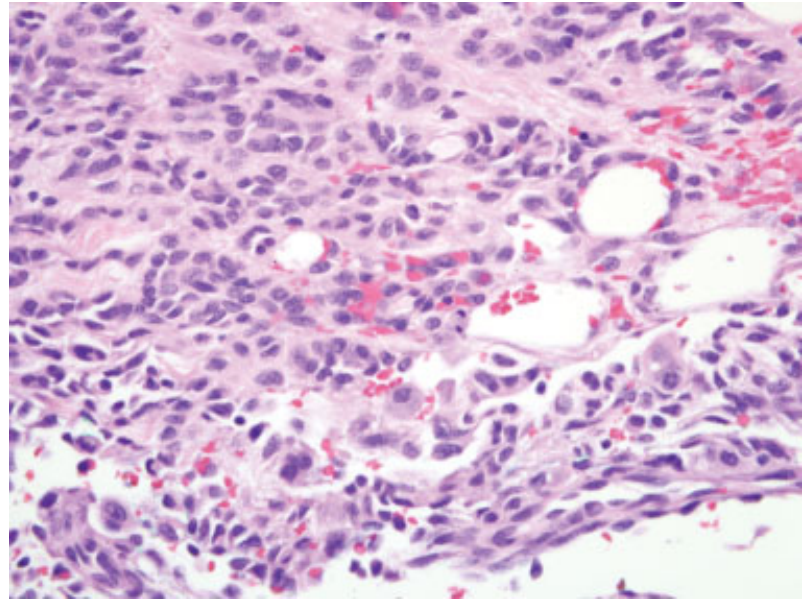

Fig. 2 Hematoxylin and eosin staining of a tumor section of patient 2 demonstrating enlarged atypical endothelial cells lining slit-like vessels ( $\times 400$ magnification). 
Table 2 Treatment and outcomes of the angiosarcoma series

\begin{tabular}{|l|l|l|l|l|l|}
\hline Case & Treatment & Margin status & Recurrence location & $\begin{array}{l}\text { Time to } \\
\text { recurrence (mo) }\end{array}$ & $\begin{array}{l}\text { Length of } \\
\text { survival (mo) }\end{array}$ \\
\hline 1 & Surgery + adjuvant radiotherapy & Positive & Left cheek & 16 & 76 \\
\hline 2 & Surgery + adjuvant radiotherapy & Positive & Right neck node & 1 & 12 \\
\hline 3 & Palliative chemotherapy & Unresectable & N/A & N/A & 60 \\
\hline 4 & Surgery & Negative & No recurrence & N/A & 53 (alive) \\
\hline 5 & Palliative radiotherapy & Unresectable & N/A & N/A & 5 \\
\hline 6 & $\begin{array}{l}\text { Neoadjuvant } \\
\text { chemoradiation }+ \text { surgery }\end{array}$ & Positive & No recurrence & N/A & 14 (alive) \\
\hline
\end{tabular}

Abbreviation: N/A, not applicable.

regional lymph node recurrence (patient 2). Two of the three patients with positive surgical margins showed recurrence (66.7\%); the 1 patient with negative margins remained recurrence-free (patient 4 ). In addition, 2 of the 3 patients treated with surgery and radiation had recurrence; the 1 patient without recurrence received neoadjuvant chemoradiation (patient 6). The patient had been recurrence free for 12 months at last follow-up on May 2013. For the series, average time to recurrence was 8.5 months, and mortality rate with recurrence was $100 \%$ (patients 1 and 2 ).

Survival rates for the series showed an overall survival and disease-specific survival rate of $33.3 \%$ ( 2 of 6 patients) and 2-year disease-free survival rate of $20 \%$ ( 1 of 5 patients) with an average survival time of 36.7 months (range of 5 to 76 months). Survival rates were then investigated based on size, grade, and postsurgical margin status. The survival rate for tumors $<5 \mathrm{~cm}$ was $50 \%$ ( 1 of 2 patients) compared with $25 \%$ for tumors $>5 \mathrm{~cm}$ ( 1 of 4 patients). Patients with low- and intermediate-grade tumors survived at the same rate as patients with high-grade tumors (33.3\%). The survival rate for positive surgical margins was $33.3 \%$ ( 1 of 3 patients) compared with $100 \%$ for negative surgical margins ( 1 patient). In addition, only 1 of 3 patients who had surgery and radiation survived (33.3\%). But because all patients who underwent radiation therapy also had positive margins, margin status was most likely a confounder. Neither palliative patient survived. The 2 patients who survived had an average age of 53.5 (52 and 57) and the 4 nonsurvivors' average age was 71.25 (range 55 to 83 ).

\section{Discussion}

Patients in our study matched the common demographics for the patient population in the literature demonstrating a mean age of 65.7 and a predilection for both males and Caucasians (both 5 to 1). Clinically, the appearance of angiosarcoma is usually rather variable. ${ }^{3}$ Similarly, patients in our series had a variety of presentations, including presenting with itching, bleeding, and pain, but the most common symptom was the presence of an enlarging nodular mass.

Surgery is historically the primary modality used to curatively treat head and neck angiosarcoma. ${ }^{3,5,6}$ The ideal treatment is to perform a wide local excision to obtain negative surgical margins. However, given the relatively small space within the head and neck, the proximity to vital structures, and the extensive microscopic subcutaneous vascular spread pattern of these tumors, it can be difficult to obtain negative margins. With the aggressive course of angiosarcoma, failing to achieve negative margins leads to high rates of local recurrence. ${ }^{4,5,7}$

The local recurrence rates after surgical excision have been reported to range from 35 to $86 \%$ in the literature. ${ }^{3,5,7-9}$ In our study, $50 \%$ of patients developed local recurrence, arising within an average of 8.5 months posttreatment. Perez et al reported similar findings, as patients who developed local recurrence after surgery and/or radiotherapy did so within 12.3 months. ${ }^{10}$ The rate of local recurrence was significantly higher with positive surgical margins. In our group of patients with positive margins, $66.7 \%$ had recurrence. Our sole patient with negative margins remains recurrence-free 53 months after treatment. Unfortunately, negative margins were only achievable in $25 \%$ of patients. This data are comparable to those of Pawlik et al, who found that their patients with positive surgical margins had a recurrence rate of $95 \%$ and only $4.7 \%$ of those with negative margins had recurrence. They also were only able to achieve negative margins in $21 \%$ of their patients. ${ }^{3}$ In view of this, it is optimal to maximize the achievable margins at the time of surgery, and a re-excision should be performed if possible for any residual disease.

The overall disease-specific survival was 33.3\%, 2-year disease-free survival was $20 \%$, and mean survival time was 36.7 months in our study. Only half of patients survived over 16 months. These poor survival rates are comparable to other studies. $^{4,5,7,10-12}$ Ogawa et al retrospectively analyzed 48 patients with angiosarcoma of the scalp and face and found a 2-year overall survival and disease-free survival rates of 22.1 and $10.7 \%$, respectively. ${ }^{7}$ Holden et al reported a 5-year overall survival of $12 \%$, with only half surviving longer than 15 months after presentation. ${ }^{2}$

Our study also suggests that tumor size and surgical margin status are significant prognostic survival factors for head and neck angiosarcoma; many studies agree. ${ }^{3,6,13}$ In regard to tumor size, Perez et al showed a 5-year overall survival rate of $48.4 \%$ for tumors less than $5 \mathrm{~cm}$ compared with $11.5 \%$ for tumors greater than $5 \mathrm{~cm} .{ }^{10}$ Our study is comparable, as our overall survival rates for tumors less than $5 \mathrm{~cm}$ and tumors greater than $5 \mathrm{~cm}$ were 50 and 25\%, respectively. For surgical margin status, our overall survival 
rate for negative and positive margins were 100 and 33.3\%, respectively. These findings are comparable to Pawlik et al, as 83.3\% of their patients with negative surgical margins survived disease-free compared with only $9.1 \%$ of those with positive surgical margins. ${ }^{3}$ Some studies also report that tumor grade is a significant prognostic factor, ${ }^{7,14}$ although others attest that there is no correlation between tumor grade and overall survival. ${ }^{3,4}$ Our results support the lack of correlation between tumor grade and survival, as the overall survival rate for patients with low- and intermediate-grade tumors was 33\%, equal to the rate for high-grade tumors.

Most studies have also reported the benefit of adjuvant radiotherapy in improving local control and increasing overall survival. $^{3-8,11,12}$ Pawlik et al reported that patients who received radiotherapy in addition to surgery survived disease-free on average 4 times longer than those treated without radiotherapy. ${ }^{3}$ However, the optimal management of radiotherapy, such as determining the optimal radiation field/dose and preventing recurrence at the margins of the field, continues to remain an issue. $^{5,15}$ Our results showed recurrence and survival rates of 66.7 and 33.3\%, respectively, among our patients who received surgery and radiation therapy. Though these rates are still concerning, all of the patients treated with curative radiation therapy in our study also had positive surgical margins. These recurrence and survival rates are slightly more favorable with adjuvant radiotherapy after tumor excision in patient populations in which both positive and negative surgical margin cases are involved. ${ }^{3,16}$

With the poor survival and local control rates of current standard therapy, physicians are searching for other treatment methods to improve survival. There are conflicting views on the role of chemotherapy as a helpful treatment modality. Although Elias and Antman found no statistically significant benefit for adjuvant chemotherapy for nonextremity angiosarcomas, more recent studies have reported benefit. ${ }^{17}$ Several reports have indicated that docetaxel and paclitaxel have demonstrated improved disease-free survival and may be effective in preventing or treating lung metastases. ${ }^{2,5,18-21}$ However, dose-limiting side effects can pose an issue. Our results also suggest a role for this combinedmodality approach as our sole survivor of positive surgical margins was treated using neoadjuvant chemotherapy (weekly paclitaxel) and radiotherapy. At last follow-up the patient was disease-free for 12 months.

Some studies have also shown benefit of the VEGFR (vascular endothelial growth factor receptors) inhibitor bevacizumab in conjunction with chemotherapy in improving quality of life and survival time in patients with distant metastases. $^{13,22}$ There also may be a role for combined bevacizumab and radiotherapy as one study reported two cases of nasal angiosarcoma remission using both modalities prior to surgical intervention, ${ }^{23}$ and another study used the combined modalities on an unresectable scalp angiosarcoma and also produced complete remission. ${ }^{6}$ Small studies have also noted similar positive findings with the VEGFR inhibitor sorafenib. ${ }^{21} \mathrm{~A}$ recent publication by Tomita et al utilized the multitargeted tyrosine kinase inhibitor (including VEGFR inhibition) pazopanib after postoperative radiotherapy and also found long-standing tumor reduction. ${ }^{24}$ Some studies have also found utility in adjuvant recombinant interleukin-2 combined with radiation therapy, suggesting that high doses of recombinant interleukin-2 helped suppress the occurrence of distant metastases. ${ }^{9}$ Further studying this method, Ohguri et al analyzed 20 patients treated with this combination and found median survival times and recurrence-free times of 36.2 and 11.1 months, respectively, similar results to our patients treated with surgery plus radiation. ${ }^{8}$

\section{Conclusion}

In conclusion, angiosarcoma is a rare vascular soft tissue sarcoma with a high rate of recurrence and a poor prognosis. Our study shows that tumor size and surgical margin status are important prognostic factors, and a multimodality treatment approach is imperative for successful outcomes. Though the optimal treatment has yet to be clearly defined, surgical resection with ideally negative margins and adjuvant radiation therapy seems to be the current mainstay. Nonetheless, based on current low rates of local control and overall survival, newer treatments such as neoadjuvant chemotherapy (especially taxane-based) and immunotherapy need further investigation to improve clinical outcomes.

\section{References}

1 Lydiatt WM, Shaha AR, Shah JP. Angiosarcoma of the head and neck. Am J Surg 1994;168(5):451-454

2 Holden CA, Spittle MF, Jones EW. Angiosarcoma of the face and scalp, prognosis and treatment. Cancer 1987;59(5):1046-1057

3 Pawlik TM, Paulino AF, McGinn CJ, et al. Cutaneous angiosarcoma of the scalp: a multidisciplinary approach. Cancer 2003;98(8): 1716-1726

4 Fury MG, Antonescu CR, Van Zee KJ, Brennan MF, Maki RG. A 14year retrospective review of angiosarcoma: clinical characteristics, prognostic factors, and treatment outcomes with surgery and chemotherapy. Cancer J 2005;11(3):241-247

5 Mark RJ, Tran LM, Sercarz J, Fu YS, Calcaterra TC, Juillard GF. Angiosarcoma of the head and neck. The UCLA experience 1955 through 1990. Arch Otolaryngol Head Neck Surg 1993;119(9): 973-978

6 De Yao JT, Sun D, Powell AT, Rehmus EH. Scalp angiosarcoma remission with bevacizumab and radiotherapy without surgery: a case report and review of the literature. Sarcoma 2011;2011:160369

7 Ogawa K, Takahashi K, Asato Y, et al. Treatment and prognosis of angiosarcoma of the scalp and face: a retrospective analysis of 48 patients. Br J Radiol 2012;85(1019):e1127-e1133

8 Ohguri T, Imada H, Nomoto S, et al. Angiosarcoma of the scalp treated with curative radiotherapy plus recombinant interleukin-2 immunotherapy. Int J Radiat Oncol Biol Phys 2005;61(5):1446-1453

9 Sasaki R, Soejima T, Kishi K, et al. Angiosarcoma treated with radiotherapy: impact of tumor type and size on outcome. Int J Radiat Oncol Biol Phys 2002;52(4):1032-1040

10 Perez MC, Padhya TA, Messina JL, et al. Cutaneous angiosarcoma: a single-institution experience. Ann Surg Oncol 2013;20(11): 3391-3397

11 Guadagnolo BA, Zagars GK, Araujo D, Ravi V, Shellenberger TD, Sturgis EM. Outcomes after definitive treatment for cutaneous angiosarcoma of the face and scalp. Head Neck 2011;33(5):661-667

12 Hodgkinson DJ, Soule EH, Woods JE. Cutaneous angiosarcoma of the head and neck. Cancer 1979;44(3):1106-1113 
13 Yang P, Zhu Q Jiang F. Combination therapy for scalp angiosarcoma using bevacizumab and chemotherapy: a case report and review of literature. Chin J Cancer Res 2013;25(3):358-361

14 Willers H, Hug EB, Spiro IJ, Efird JT, Rosenberg AE, Wang CC. Adult soft tissue sarcomas of the head and neck treated by radiation and surgery or radiation alone: patterns of failure and prognostic factors. Int J Radiat Oncol Biol Phys 1995;33(3):585-593

15 Morrison WH, Byers RM, Garden AS, Evans HL, Ang KK, Peters LJ. Cutaneous angiosarcoma of the head and neck. A therapeutic dilemma. Cancer 1995;76(2):319-327

16 Mark RJ, Poen JC, Tran LM, Fu YS, Juillard GF. Angiosarcoma. A report of 67 patients and a review of the literature. Cancer 1996; 77(11):2400-2406

17 Elias AD, Antman $\mathrm{KH}$. Adjuvant chemotherapy for soft tissue sarcoma: an approach in search of an effective regimen. Semin Oncol 1989;16(4):305-311

18 Nagano T, Yamada Y, Ikeda T, Kanki H, Kamo T, Nishigori C. Docetaxel: a therapeutic option in the treatment of cutaneous angiosarcoma: report of 9 patients. Cancer 2007;110(3):648-651
19 Isogai R, Kawada A, Aragane Y, Tezuka T. Successful treatment of pulmonary metastasis and local recurrence of angiosarcoma with docetaxel. J Dermatol 2004;31(4):335-341

20 Karube R, Sasaki H, Shinozuka K, et al. Angiosarcoma of the scalp diagnosed by the presence of neck inflammation: a case report. Int J Oral Sci 2012;4(3):166-169

21 Singla S, Papavasiliou P, Powers B, et al. Challenges in the treatment of angiosarcoma: a single institution experience. Am J Surg 2014; 208(2):254-259

22 Fuller CK, Charlson JA, Dankle SK, Russell TJ. Dramatic improvement of inoperable angiosarcoma with combination paclitaxel and bevacizumab chemotherapy. J Am Acad Dermatol 2010;63(4): e83-e84

23 Koontz BF, Miles EF, Rubio MA, et al. Preoperative radiotherapy and bevacizumab for angiosarcoma of the head and neck: two case studies. Head Neck 2008;30(2):262-266

24 Tomita H, Koike Y, Asai M, et al. Angiosarcoma of the scalp successfully treated with pazopanib. J Am Acad Dermatol 2014; 70(1):e19-e21 